\title{
Study on optical finite impulse response filter
}

\author{
Ying Zhou \\ Guangjie Zeng \\ Feihong Yu \\ Zhejiang University \\ State Key Laboratory of Modern Optical \\ Instrumentation \\ Optical Engineering Department \\ Hangzhou 310027 China \\ E-mail: feihong@zju.edu.cn \\ H. S. Kwok \\ Hong Kong University of Science and \\ Technology \\ Center for Display Research and \\ Department of Electronic and Electrical \\ Engineering \\ Clear Water Bay, Kowloon \\ Hong Kong
}

\begin{abstract}
We present an optical finite impulse response (FIR) filter design method. Based on crystal birefringence, an optical FIR filter can be designed to produce arbitrary spectrum output. A mathematic model of the optical FIR filter is introduced from the FIR theory. Details of the mapping relationship between an FIR filter and an optical filter are analyzed. A classical FIR filter design method is directly applied. After using the optical backward transfer method to implement the optical configuration, a design of a green/magenta broadband filter is discussed. The experiment results demonstrate that the theoretical simulation is in accordance with the experiment. (C) 2003 Society of Photo-Optical Instrumentation Engineers. [DOI: 10.1117/1.1586289]
\end{abstract}

Subject terms: finite impulse response filter; optical finite impulse response filter; crystal birefringence; extended Jones matrix; arbitrary spectrum output.

Paper 020542 received Dec. 13, 2002; revised manuscript received Jan. 22, 2003; accepted for publication Jan. 22, 2003.

\section{Introduction}

An optical filter is one of the most important devices used in optical system spectrum crunching and optical signal processing. The present optical filters mainly consist of two broad categories: thin film filter and birefringent filter. There are several types of filters based on the latter, such as multiple tunable liquid crystal filters, ${ }^{1}$ Lyot filters, and Solc filters. ${ }^{2}$ Birefringent filters have been used widely, such as in the observation of solar prominence, selection of laser wavelength, separation of wavelength signals, endoscope imaging, remote sensing, optical communication, etc. ${ }^{3}$

It has been a long time since the idea of a filter comprised of cascaded birefringent retarders appeared, but the specific outputs it produces limit its wider applications to some extent. Although the multiple tunable liquid crystal filter makes use of electrooptic effects to obtain the desired spectrum output, it still can be simplified as a series of retardation plates in a continuous rotation. However, it can be difficult to control due to the influences of temperature, viewing angle, material stress, sandwiched structure, and other outside circumstances. The Lyot and Solc filters have strong regularities in structure: the retarders in Lyot filters are ranged in the same rotation angles but in geometric series thicknesses, while the retarders of identical thicknesses in Solc filters are disposed in a folded or sectorshaped rotation. This structural regularity results in simple spectrum output because of the freedom constraint, so usually only narrowband output is available. However in some other optical processing cases, such as projection display and color manipulation, a filter that is able to provide an arbitrary spectrum is required. This is a big challenge for the capabilities of conventional birefringent filters.

Also based on crystal birefringence, an optical finite impulse response (FIR) filter is comprised of a series of crystal retarders between two polarizers (or a polarization beamsplitter). In contrast, it manages to produce an arbitrary spectrum of two complementary colors orthogonal with each other. In this work, we concentrate on how to realize optical FIR filters producing arbitrary desired spectrums. The classical FIR theory in electronics is used to establish the mathematical model of this optical FIR filter and the numerical approximation method is employed in the filter design to search the suitable coefficients of the output series. Once the coefficients are obtained, they can be used to determine the real orientation of each retarder by optical backward transfer techniques. Through the example discussed, we can see that the main merits of this filter are the convenience of design, good flexibility, and good performances of both spectrum and angle properties.

\section{Model of the Optical FIR Filter}

\subsection{Mathematical Model of the Optical FIR Filter}

In order to see the correspondence of a FIR filter with an optical FIR filter, we give a brief depiction of the FIR theory. For a discrete linear system and a causal system, the output and input of a digital filter could be described by a convolution in the time domain. It is shown in Eq. (1) as ${ }^{4}$ :

$y(n)=\sum_{k=-\infty}^{\infty} h(k) x(n-k)$

where $x(n)$ is a discrete time series and $h(k)$ is the response of a unit sampling series $\delta(n)$. If $h(k)$ includes finite sampling dots only in the range $0 \leqslant k \leqslant N-1$ ( $N$ is finite), the system is called a finite impulse response digital filter (FIR filter). The frequency response of this FIR filter can be easily obtained though the Fourier transform.

$H[\exp (i \omega T)]=\sum_{k=0}^{N-1} h(k) \exp (-i k \omega T)$. 


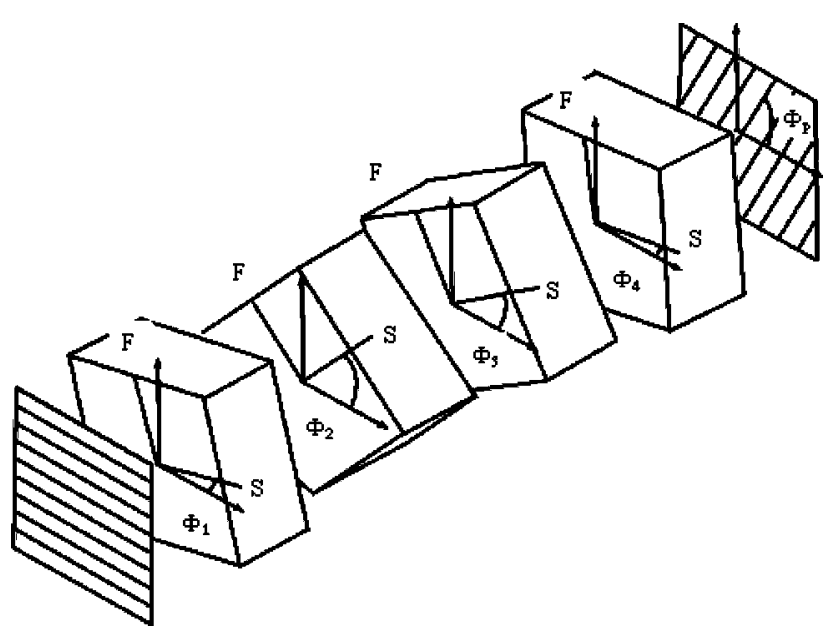

Fig. 1 The general structure of an optical FIR filter.

Obviously, in the frequency field a FIR filter is the sum of a finite Fourier series, and the coefficients of the Fourier series are unit impulse responses. It corresponds with an infinite discrete series distributed on the whole time axis. But to make it realizable, the approximation is made by intercepting finite terms and shifting in the meantime. So how to intercept and revise coefficients is the key problem in FIR filter design.

Figure 1 shows the general structure of optical FIR filter composed of a series of birefringent retarders: $n$ birefringent crystal retarders of identical length and material are cascaded at specific rotation angles between two polarizers with the optical axis of each retarder parallel to its optical surface. The anisotropism in the crystal leads to the separation of o-light and e-light with different velocities. When linear polarized light comes through the retarder, it is divided into two components along the fast-axis and lowaxis, respectively, and they serve as the input of the second plate. The output of the second retarder can be combined into three different impulses in a time series along a fastfast axis, fast-slow axis, and slow-slow axis, respectively. So it can be learned by analogy that such an optical network as $n$ crystal retarders chosen with the same material and length will definitely produce $n+1$ impulses with the identical time interval, ${ }^{5}$ described by Eq. (3).

$$
\begin{aligned}
C(t)= & C_{0} \delta(t)+C_{1} \delta(t-a)+C_{2} \delta(t-2 a)+\cdots \\
& +C_{n} \delta(t-n a) \\
= & \sum_{k=0}^{n} C_{k} \delta(t-k a),
\end{aligned}
$$

where $a$ denotes the time interval of the impulse series, given by

$a=t_{s}-t_{f}=L \Delta \eta / c$,

where $t_{s}, t_{f}$ denote the time needed to transit a single retarder of the two impulses, $L$ is the length of each retarder, and $\Delta \eta$ denotes the difference of refraction indices:

$\Delta \eta=n_{e}-n_{o}$.
Here $c$ is the light velocity in a vacuum. Thus the phase difference caused by a single retarder is determined as

$\Gamma=\frac{2 \pi \Delta \eta L}{\lambda}$

where $\lambda$ is the wavelength in a vacuum. The Fourier transform (FT) of Eq. (3) is expressed as:

$$
\begin{aligned}
C(\omega)= & C_{0}+C_{1} \exp (-i a \omega)+C_{2} \exp (-i 2 a \omega)+\cdots \\
& +C_{n} \exp (-i n a \omega) \\
= & \sum_{k=0}^{n} C_{k} \exp (-i k a \omega) .
\end{aligned}
$$

Thus we know that the frequency output of an optical FIR filter is a Fourier series with finite terms and real coefficients, coincident with a standard FIR filter. In this sense, such an optical linear network could be termed as a FIR filter realized by optics. Its physical parameters are mapped into the parameters of the FIR filter. For example, the optical impulse output is in correspondence with the impulse series of an FIR filter. Its frequency response through the Fourier transform approximates the desired output.

Now we restate Eq. (7) into two other forms, which are totally equivalent:

$$
\begin{aligned}
C(\omega)= & C_{0}+C_{1} \exp (-i \Gamma)+C_{2} \exp (-i 2 \Gamma)+\cdots \\
& +C_{n} \exp (-i n \Gamma) \\
= & \sum_{k=0}^{n} C_{k} \exp (-i k \Gamma), \\
C(\lambda)= & C_{0}+C_{1} \exp \left[-i 2 \pi \Delta \eta L\left(\frac{1}{\lambda}\right)\right] \\
& +C_{2} \exp \left[-i 22 \pi \Delta \eta L\left(\frac{1}{\lambda}\right)\right]+\cdots \\
& +C_{n} \exp \left[-i n 2 \pi \Delta \eta L\left(\frac{1}{\lambda}\right)\right] \\
= & \sum_{k=0}^{n} C_{k} \exp \left[-i k 2 \pi \Delta \eta L\left(\frac{1}{\lambda}\right)\right]
\end{aligned}
$$

where $\omega=2 \pi f=2 \pi c / \lambda$ represents the angle frequency of the light wave. It changes the target function (nonperiod in spectrum) into the phase function (period function) to map the optical filter into a standard FIR filter. Here period $2 \pi$ corresponds with the Nyquist sampling frequency.

Theoretically the ideal frequency response $C_{d}(\omega)$ could be developed precisely as an infinite Fourier series, which corresponds with infinite crystal retarders. Therefore, like the FIR filter, how to choose the FT coefficients to approximate the desired output and how to select $n$ retarders to concatenate in a proper way are the key problems in optical FIR filter design.

The simplest method is to make a direct truncation of $n$ FT terms, but the Gibbs phenomenon resulting from a side lobe convolution of the rectangular window is extremely 


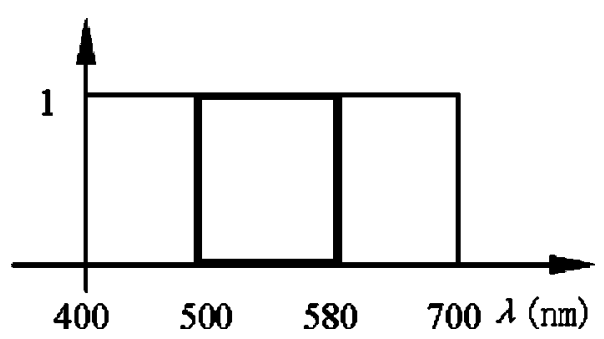

Fig. 2 Spectrum output of desired G/M filter.

heavy. The fewer the terms, the larger the ripples in the passband and stop band, and the broader the transition band. However, with the number of terms increasing, overshoot and undershoot will appear at the break points despite better approximation. So the ripples and transition band restrict each other no matter how many terms there are. At a given number of terms, we can only make a compromise between them to meet our needs. The window function does well in reducing ripples but it costs the transition band. In our design, we use a numerical approximation algorithm (Chebyshev and least-square filter design algorithms) for this optical FIR filter design. ${ }^{4,6}$

\subsection{FIR Filter Design for the Optical FIR Filter}

Assuming that $C_{d}(\omega)$ is the desired spectrum and $C(\omega)$ is the actual spectrum, the Chebyshev approximation gives the design satisfying:

$\min \left(\int\left\{W(\omega)\left[\left|C_{d}(\omega)\right|-C(\omega)\right]\right\}^{p} d \omega\right)$,

where $W(\omega)$ is the weight of each band. It means that at a given weight, this algorithm will give the optimal filter in the sense that the maximal error is minimal. It shows equiripple property in the passband and stop band. When $p=2$, it turns into the least-square approach. In this way we can balance the ripples and transition band quite effectively, and even get specific responses in specific frequency points through parameter control.

Here we give an example of a green/magenta $(\mathrm{G} / \mathrm{M})$ broadband filter used in the optical engine of a projection display. Figure 2 shows the desired spectrum response of the $\mathrm{G} / \mathrm{M}$ filter. The thick line represents the green output and the thin line represents the magenta output.

Figure 3 shows the simulated spectrum of an optical FIR filter using Chebyshev and least-square approaches $\left(C_{k}\right.$ obtained from these two approaches can be found in Table 1) for the 10 order filter concerned and the phase delay of each plate $\Delta \eta L=805 \mathrm{~nm}$. The spectrum is the normalized distribution of $C^{2}(\lambda)$ as light intensity $[C(\lambda)$ is constructed by Eq. (9)].

The equiripple design with the Chebyshev approach reaches ripples no larger than $2 \%$, while the result with the least-square approach reaches the ripples no more than $1 \%$, but has a little broader transition band.

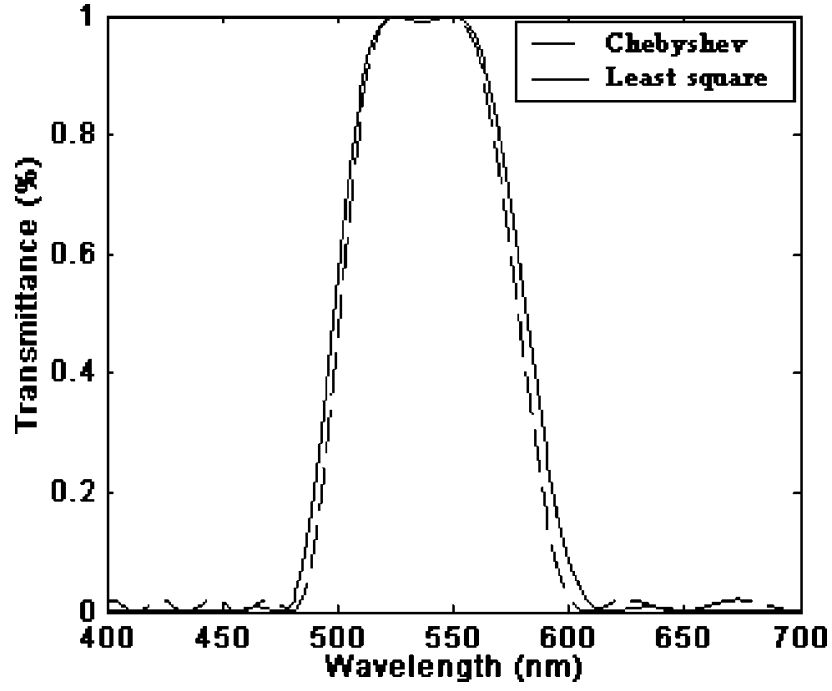

Fig. 3 G/M filter designed by Chebyshev and least-square approaches.

\section{Optical Implementation of the Optical FIR Filter}

\subsection{Determination of Physical Parameters for the Optical FIR Filter}

Once the series of impulse responses is obtained, we can use optical backward transfer synthesis techniques proposed by Harris to implement the filter in optics. ${ }^{5}$

Suppose that the output component along the direction of the analyzer is expressed in Eq. (7) and the perpendicular output is denoted as $D(\omega)$. Being the same form as $C(\omega)$, it forms the complementary color of $C(\omega)$.

$$
\begin{aligned}
D(\omega)= & D_{0}+D_{1} \exp (-i a \omega)+D_{2} \exp (-i 2 a \omega)+\cdots \\
& +D_{n} \exp (-i n a \omega) \\
= & \sum_{k=0}^{n} D_{k} \exp (-i k a \omega) .
\end{aligned}
$$

\begin{tabular}{|c|c|c|c|c|c|c|}
\hline \multirow[b]{2}{*}{$k$} & \multicolumn{3}{|c|}{$\begin{array}{l}\text { Least-square } \\
\text { approach design }\end{array}$} & \multicolumn{3}{|c|}{$\begin{array}{c}\text { Chebyshev } \\
\text { approach design }\end{array}$} \\
\hline & $C_{k}$ & $D_{k}$ & $\begin{array}{c}\theta_{k} \\
\text { (in radius) }\end{array}$ & $C_{k}$ & $D_{k}$ & $\begin{array}{l}\theta_{k} \text { (in } \\
\text { radius) }\end{array}$ \\
\hline 1 & 0.0476 & -0.6560 & -1.4983 & 0.0785 & -0.6925 & -1.4579 \\
\hline 2 & -0.0195 & -0.5260 & -0.0872 & 0.0129 & -0.5255 & -0.0665 \\
\hline 3 & -0.0522 & 0.2280 & 0.0153 & -0.0746 & 0.2517 & -0.0156 \\
\hline 4 & 0.1490 & -0.0278 & 0.1766 & 0.1560 & -0.0560 & 0.1969 \\
\hline 5 & -0.2328 & -0.0686 & -0.4427 & -0.2251 & -0.0519 & -0.4295 \\
\hline 6 & 0.2660 & 0.0846 & 0.5811 & 0.2521 & 0.0850 & 0.5462 \\
\hline 7 & -0.2328 & -0.0580 & -0.4427 & -0.2251 & -0.0706 & -0.4295 \\
\hline 8 & 0.1490 & 0.0237 & 0.1766 & 0.1560 & 0.0385 & 0.1969 \\
\hline 9 & -0.0522 & -0.0013 & 0.0153 & -0.0746 & -0.0105 & -0.0156 \\
\hline 10 & -0.0195 & -0.0056 & -0.0872 & 0.0129 & -0.0038 & -0.0665 \\
\hline 11 & 0.0476 & 0.0035 & 0.0725 & 0.0785 & 0.0089 & 0.1129 \\
\hline
\end{tabular}

Table 1 Numerical results of optical backward transfer techniques for two approaches. 


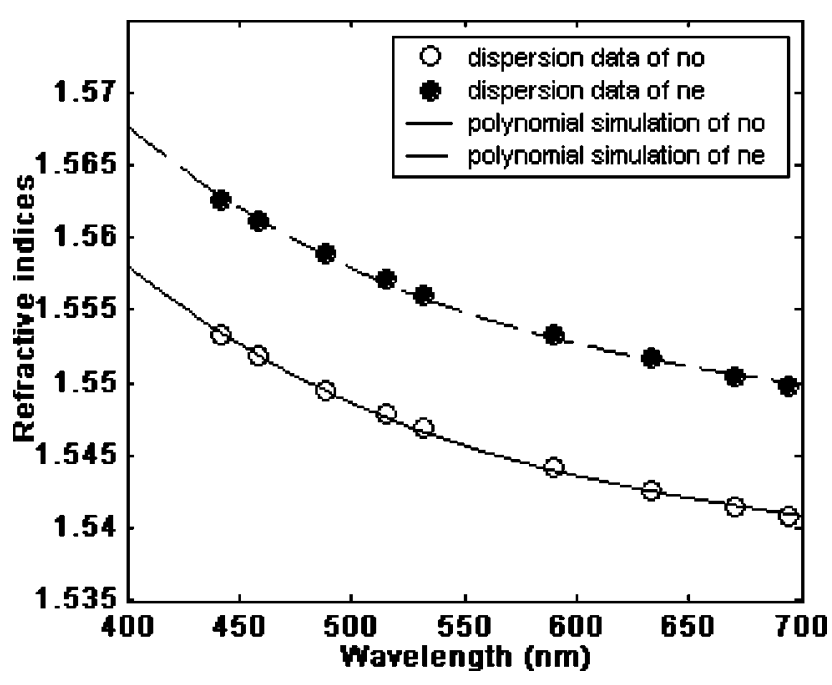

Fig. 4 Simulation of quartz dispersion.

When ignoring the crystal absorption, the two components satisfy the principle of energy conservation.

$D(\omega) D(\omega)^{*}=I_{0}^{2}-C(\omega) C(\omega)^{*}$,

where $I_{0}^{2}$ is the intensity of the incident light. It can be chosen to be an arbitrary value as long as the two components are both nonnegative. It has been demonstrated that there exist more than one set of real solutions under certain constraints, noted as $C_{k}$ and $D_{k}$. Each set gives a complete description of the output. Usually we could find $2^{(n-m / 2+1)}$ ( $n$ is the number of retarders and $m$ is the number of complex solution) sets of $D_{k}$ and $C_{k}$ corresponding with a specific $I_{0}^{2}$, therefore, we get $2^{(n-m / 2+1)}$ sets of different angles to produce the same output. That is to say, they are not independent of each other and have some redundancies.

In the optical system shown in Fig. 1, the direction of the polarizer is chosen to be along the $x$ axis. Rather than

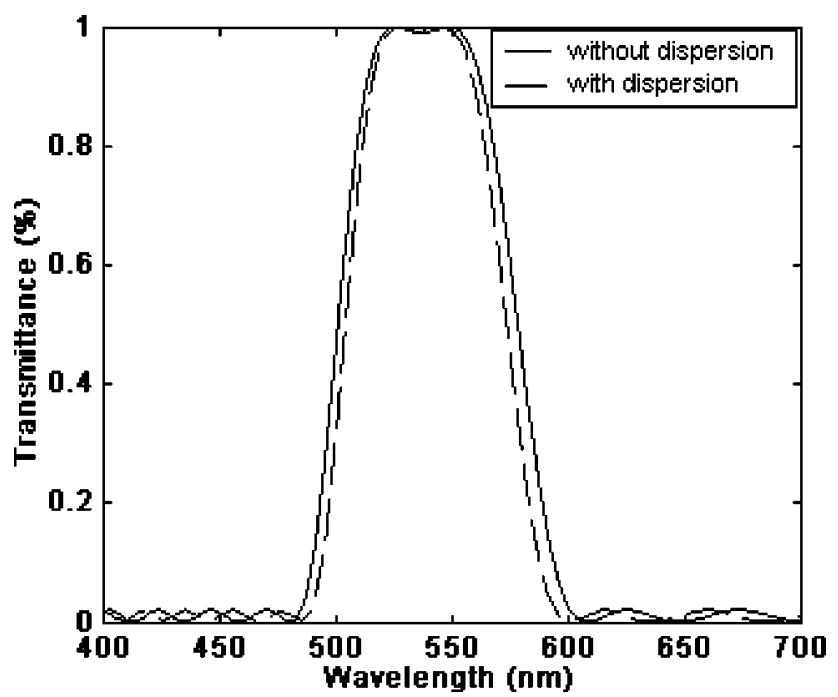

Fig. 5 Chebyshev-based G/M filter design with dispersion.

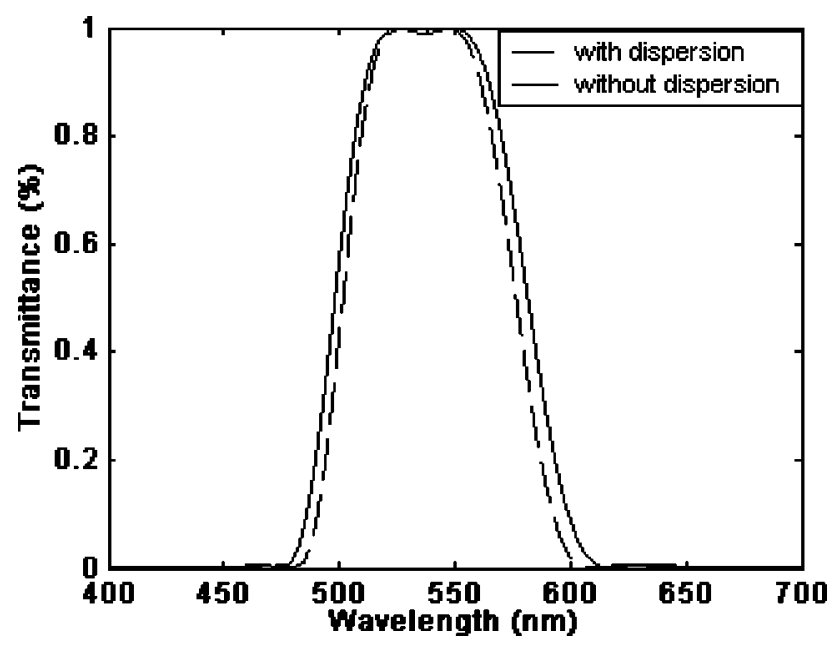

Fig. 6 Least-square-based G/M filter design with dispersion.

dealing with absolute angles of each crystal retarder relative to $x, \phi_{1}, \phi_{2}, \ldots, \phi_{n}$ and $\phi_{p}$ (the direction of the analyzer), we define

$\theta_{1}=\phi_{1}, \quad \theta_{2}=\phi_{2}-\phi_{1}, \ldots, \theta_{n}=\phi_{n}-\phi_{n-1}$,

$\theta_{p}=\phi_{p}-\phi_{n}$

as the relative angles of each retarder. The whole set of angles could be determined from rear to front with the optical backward transfer synthesis technique by virtue of the relationship between the input and output of each retarder and its coordinates. ${ }^{5,7}$ Hence the spectrum could be physically determined by the Jones matrix. ${ }^{8}$ Suppose that the Jones matrix of polarized impulses behind the polarizer is $E_{0}=\left[\begin{array}{l}1 \\ 0\end{array}\right]$, the output is:

$$
\begin{aligned}
E_{\text {out }}= & {\left[\begin{array}{cc}
\cos \theta_{p} \exp (-i \Gamma) & \sin \theta_{p} \\
-\sin \theta_{p} \exp (-i \Gamma) & \cos \theta_{p}
\end{array}\right] } \\
& \times \prod_{i=2}^{n}\left[\begin{array}{cc}
\cos \theta_{i} \exp (-i \Gamma) & \sin \theta_{i} \\
-\sin \theta_{i} \exp (-i \Gamma) & \cos \theta_{i}
\end{array}\right]\left[\begin{array}{c}
\cos \theta_{1} \\
-\sin \theta_{1}
\end{array}\right] .
\end{aligned}
$$

For the two results given in Fig. 3, the corresponding $C_{k}, D_{k}$, and $\theta_{k}$ determined by the least-square and Chebyshev approaches are listed in Table 1 . Here $\theta_{11}=\theta_{p}$ is the orientation of the analyzer.

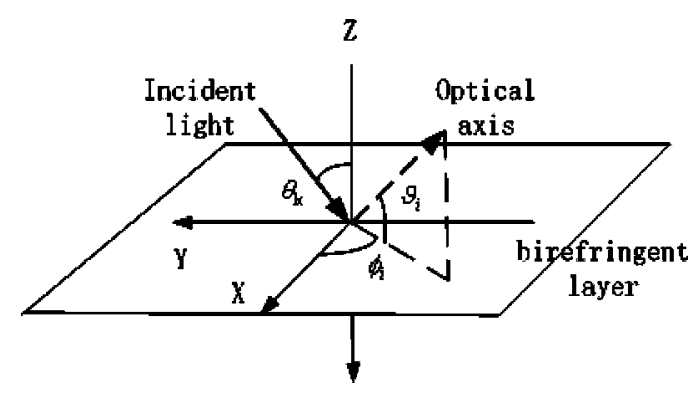

Fig. 7 Coordinate of a birefringent layer in an extended Jones matrix. 


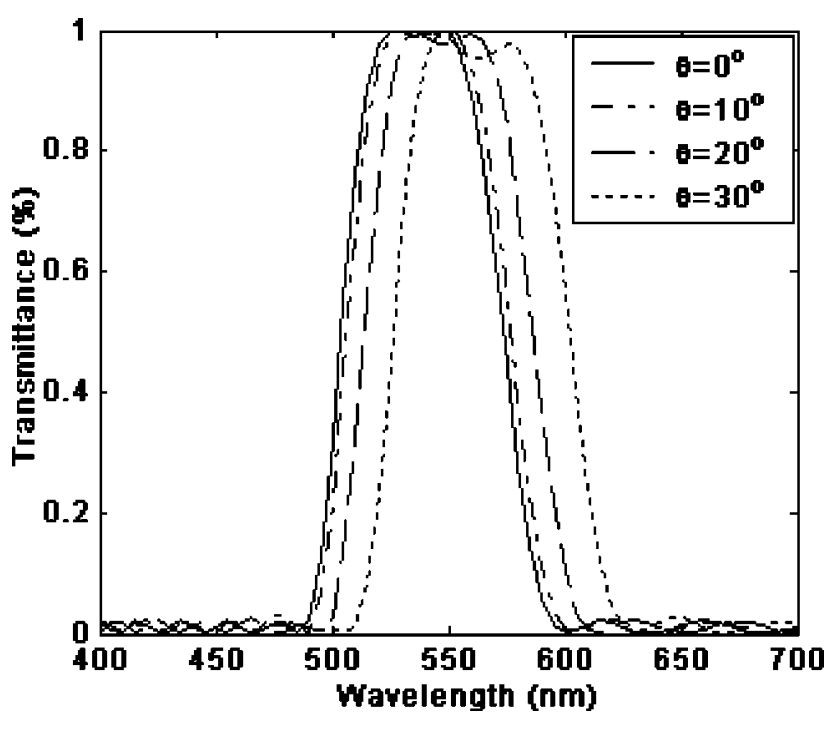

Fig. 8 Angle property of design with the Chebyshev approach.

\subsection{Angle Property Evaluation of the Filter Considering Material Dispersion}

The material of the crystal plate used in the experiment is quartz. Considering the material dispersion, the refractive indices of quartz $n_{o}$ and $n_{e}$ are simulated by a 3 order polynomial for each one, shown in Fig. 4. The impact of material dispersion to the original design is shown in Figs. 5 and 6 , respectively. It can be seen that the spectrum is slightly narrowed by dispersion symmetrical to the central wavelength $(540 \mathrm{~nm}$ for $\mathrm{G} / \mathrm{M}$ filter).

To find the performance of this filter under oblique incidence, we employ the extended Jones matrix ${ }^{9,10}$ to evaluate the angle property of the optical FIR filter. An extended Jones matrix provides a useful tool to treat the transmittance of off-axis light in a general birefringent network (optical axis of a birefringent layer in an arbitrary direction). In the coordinate defined in Fig. 7, the $x y$ plane represents a birefringent layer. When employing the extended

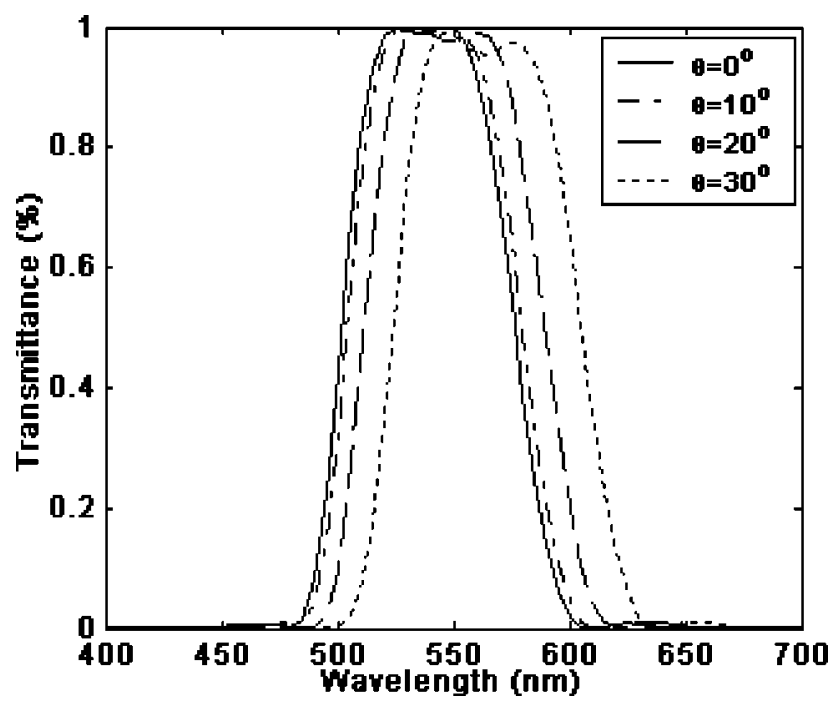

Fig. 9 Angle property of design with the least-square approach.

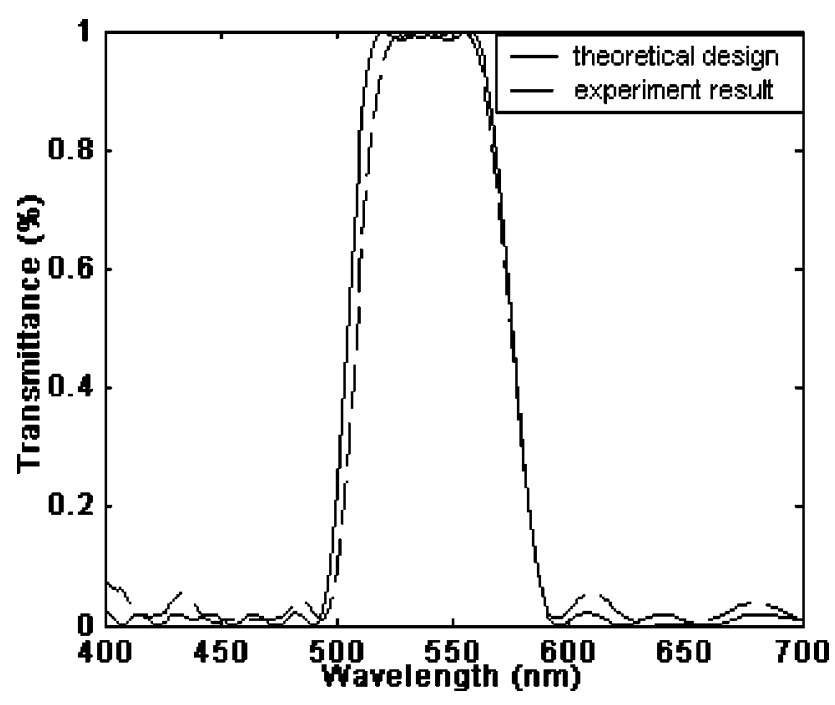

Fig. 10 Experiment result for an optical FIR filter: spectrum property.

Jones matrix, we just have to force the azimuth angle of the optical axis in the $x y$ plane, which means $\vartheta_{i}=0$ for each plate. ${ }^{9}$ The simulation results of the angle property by the Chebyshev and least-square approach designs are shown in Figs. 8 and 9, respectively (in consideration of the dispersion).

Within \pm 20 -deg oblique incidence, the curve shows little distortion but only a certain parallel shift. Both of these two filters show good performances of angle property. The curve within \pm 5 -deg incidence is almost the same as the curve under normal incidence.

\section{Experiment Results}

Since the error of the filter comes mainly from the relative orientations of the retarders, and the error increases as the number of retarders increases, we present the experiment of another design result, which is practically used in our liquid crystal on silicon (LCOS) projection display system, for

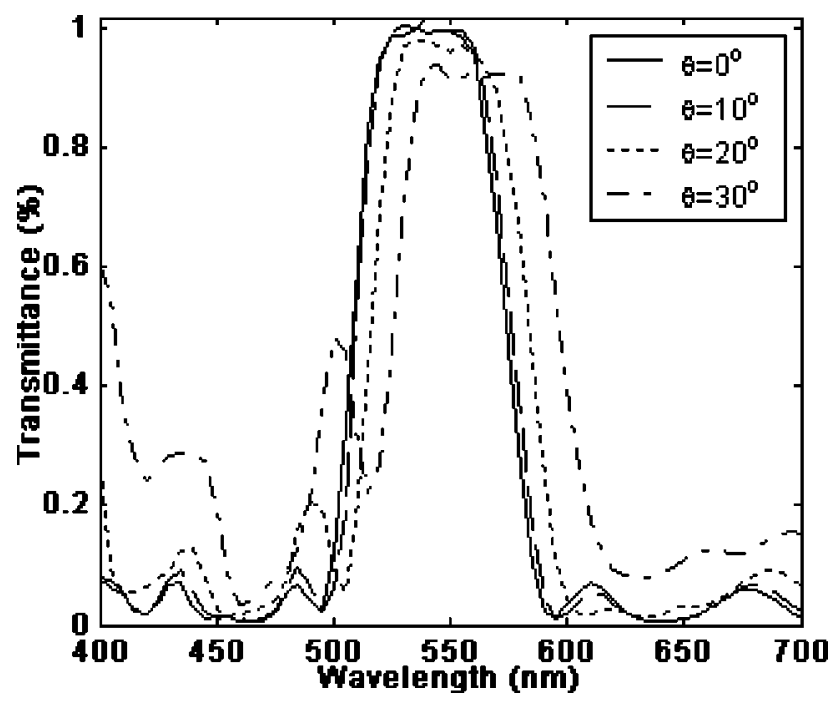

Fig. 11 Experiment angle property of the optical FIR filter. 


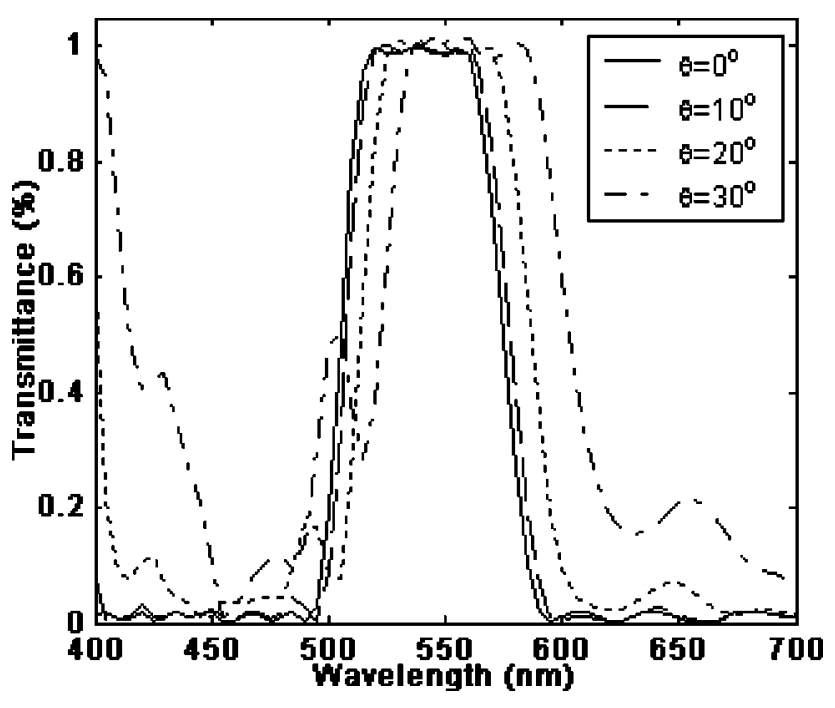

Fig. 12 Theoretical simulation of angle property.

nine plates and $\Delta \eta d=1075 \mathrm{~nm}$. The incident light is collimated. The normalized spectrum waves scanned by the spectrometer for normal incidence and for oblique incidence are shown in Figs. 10 and 11, respectively. The simulated angle property is shown in Fig. 12. Due to the simple manual process, the experiment shows differences mainly in the ripples between the experimental and theoretical results. However, these figures demonstrate that the characteristics of these rudimentary experiment results of the optical FIR filter agree quite well with our theoretical simulation.

\section{Conclusions}

A FIR filter theory is employed to make an optical filter design with a structure of cascaded birefringent crystal retarders. From the mathematical model, the given algorithms, and the design procedure, we succeed in realizing an arbitrary spectrum output design, which is much less restricted. Not only does it provide complementary colors in double channels (through 90-deg rotation of the analyzer), but it has excellent wide-angular properties as well. As a result, this will contribute greatly to a more compact optical system and higher energy efficiency. The example presented here acts as a color separation/combination component of an optical engine in LCOS projection display with a very simple structure. Therefore, this optical FIR filter is of great value in practical use. ${ }^{11,12}$

\section{Acknowledgments}

This project is supported by the National Natural Science Foundation of China, and the Joint Foundation of Ningbo Science and Technology Commission and Zhejiang University.

\section{References}

1. Y. Kong, G. H. Li, M. J. Yun, X. Y. Fan, Y. T. Pang, and N. Wang, “A research on multiple tunable liquid crystal filters," J. Optoelectron. Laser 12(5), 474-476 (2001).

2. Alev and P. Yeh, Optical Waves in Crystals: Propagation and Control of Laser, Science Press, Beijing (1991)

3. Z. X. Liang, G. H. Li, W. D. Shao, and X. Wang, "Study of optimal thickness ratio of birefringent crystals," Chin. J. Lasers 27(7), 601$605(2000)$.

4. Z. Y. He, Digital Signal Processing: Theory and Application, People's Posts \& Telecommunications Press, Beijing (1983).

5. S. E. Harris, E. O. Ammann, and I. C. Chang, "Optical network synthesis using birefringent crystals. I. Synthesis of lossless network of equal-length crystals," J. Opt. Soc. Am. 54(10), 1267-1279 (1964).

6. G. S. Hu, Digital Signal Processing-Theory, Algorithm and Realization, Tsinghua University Press, Beijing (1997).

7. G. D. Sharp and J. R. Birge, "Retard stack technology for color manipulation," SID Symp. 30, 1072-1075 (1999).

8. Y. B. He, J. Y. Xiong, F. H. Yu, and W. Zheng, "Application of Jones matrix in design of polarization interference filter," Acta Photonica Sinica 31(4), 507-512 (2002)

9. A. Lien, "Extended Jones matrix representation for the twisted nematic liquid-crystal display at oblique incidence," Appl. Phys. Lett. 57(26), 2767-2769 (1990).

10. F. H. Yu and H. S. Kwok, "Comparison of extended Jones matrices for twisted nematic liquid crystal displays at oblique angles of incidence," J. Opt. Soc. Am. A 16(11), 2772-2780 (1999).

11. X. J. Yu, Y. B. He, J. Y. Xiong, F. H. Yu, and H. S. Kwok, "Design of polarization interference filters," Displays 23, 145-149 (2002).

12. Y. B. He, J. Y. Xiong, W. Zheng, G. J. Zeng, and F. H. Yu, "Technology and applications of LCOS projector," Opt. Instruments 24(2), $38-46(2002)$

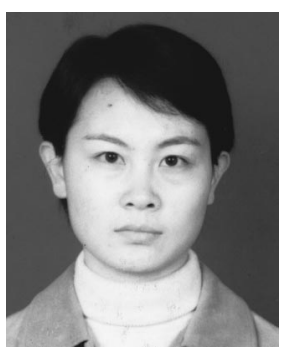

Ying Zhou is a graduate candidate of the optical engineering department at Zhejiang University, China. She received her BS in 2000 also from the optical engineering department of Zhejiang University. Her research interests are mainly crystal optics, optical signal processing, and display devices.

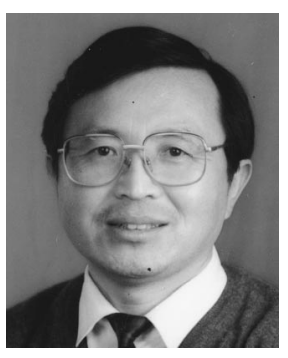

Guangjie Zeng graduated from Zhejiang University in 1976. His research interests are laser and spectrum techniques.

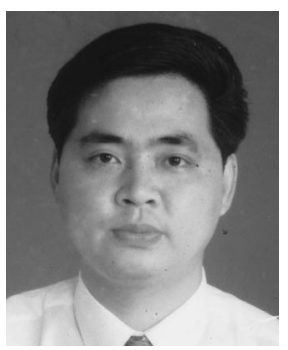

Feihong Yu received his $\mathrm{PhD}$ degree from the optical engineering department at Zhejiang University in 1993. His main interests are liquid crystal displays, LCD projection display, optical and digital image processing, optical instrumentation, and 3-D medical image processing and visualization. $\mathrm{He}$ is the author or coauthor of more than 90 technical papers. 